\title{
Investigation of methods for the formation of multicolored images reconstructed with protective holograms
}

\author{
L.A. Nayden ${ }^{1}$, I.K. Tsyganov ${ }^{1}$, S.B. Odinokov ${ }^{1}$ \\ ${ }^{I}$ Moscow State Technical University named after N. U. Bauman, ul. Baumanskaya 2-ya, 5, 105005, Moscow, Russia
}

\begin{abstract}
Dot-matrix holograms contain diffraction gratings with different periods and orientations. Grating parameters (period and orintation) are calculated according to input data, which is graphic raster file. Traditionally image uses additive color model RGB, which involves limited color range. To increase color range International Illumination Commission (ICI) color model is considered. In this paper methods for calculating the parameters of diffraction gratings with different periods and orientations for ICI graphic files are investigated and analyzed.
\end{abstract}

Keywords: Diffraction gratings; Colored holograms; Color chart; Dot-Matrix; Colorimetric system

\section{Introduction}

Result of the calculation dot-matrix hologram is a set of diffraction gratings. Color, displayed from hologram pixel, should be close to the color of the corresponding pixel of the input image. The following grating parameters are used to set hologram pixel color:

- The period of the holographic grating;

- Angular orientation of the holographic grating;

- Relief parameters - depth and profile type.

\section{Methods for calculating holographic pixel parameters}

The period of the holographic diffraction grating determines the wavelength of the radiation diffracted on it. The the varying grating orientation angular orientation determines the angle of rotation when a certain pattern is restored. It is possible to achieve a smooth color change in the image when the hologram is rotated. Relief parameters: namely the depth and type of the profile, which determine the brightness of the radiation diffracted from a particular pixel. The brightness of diffracted radiation is determined by profile depth and type and grating area. The parameters of the diffraction gratings can be simply described in the HSB colorimetric system, where the grating period corresponds to the Hue coordinate, and the brightness of the diffracted radiation corresponds to the coordinates of the Saturation and Brightness.

The original image for rainbow holograms in most cases can be created using image editors on the computer. The result is a raster image file, in which the image is represented as a finite set of pixels [2]. Image dot form a graphic pixel with diffraction gratings located at a very small distance from each other. The perceived color of a pixel is coming result of diffraction in different holographic gratings. Pixels contain information about the color described in the RGB colorimetric system [3-4]. There are known formulas for converting color coordinates between colorimetric systems HSB and RGB. This fact allows us to calculate the parameters of holographic diffraction gratings from the input image file.

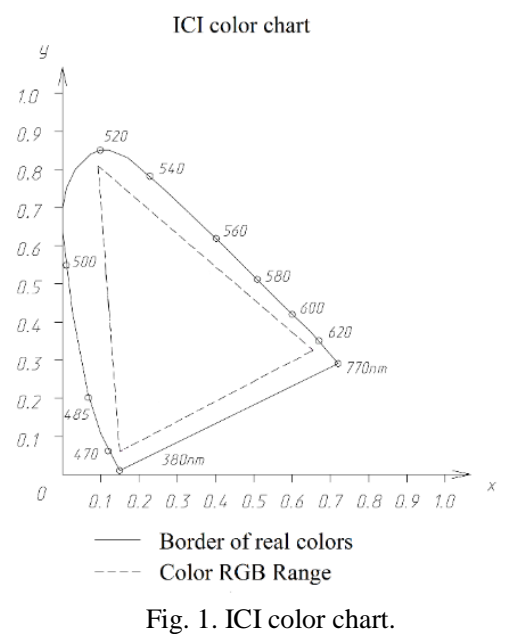

Although color acquisition using the RGB system is widely used in many areas, and the RGB system can be used display a wide range of colors, but it still can not cover all possible colors in the ICI chart. To expand color range reproduction by means 
of diffraction gratings, method of color reconstructing in the ICI colorimetric system (1931) is used. The ICI and RGB colormaps are illustrated on Fig. 1.

Any three different diffraction dots representing three different wavelengths can form any color within the ICI diagram. X, Y, $\mathrm{Z}$ are the color coordinates, the vertices of the triangle in which the color is formed. According to the ICI theory, as shown in Fig. 2, the required color is the color having the coordinates $\mathrm{x} 0 \mathrm{y} 0$ and the intensity V0 denoted by [(x0, y0), Y0]. The three selected points have the following coordinates (x1 y1), (x2 y2), (x3 y3). The intensity of the diffracted light at these three points of light will be denoted by Y1, Y2, Y3. Then, knowing the coordinates of these points, through non-complex calculations, one can come to the definition of the required coordinate.

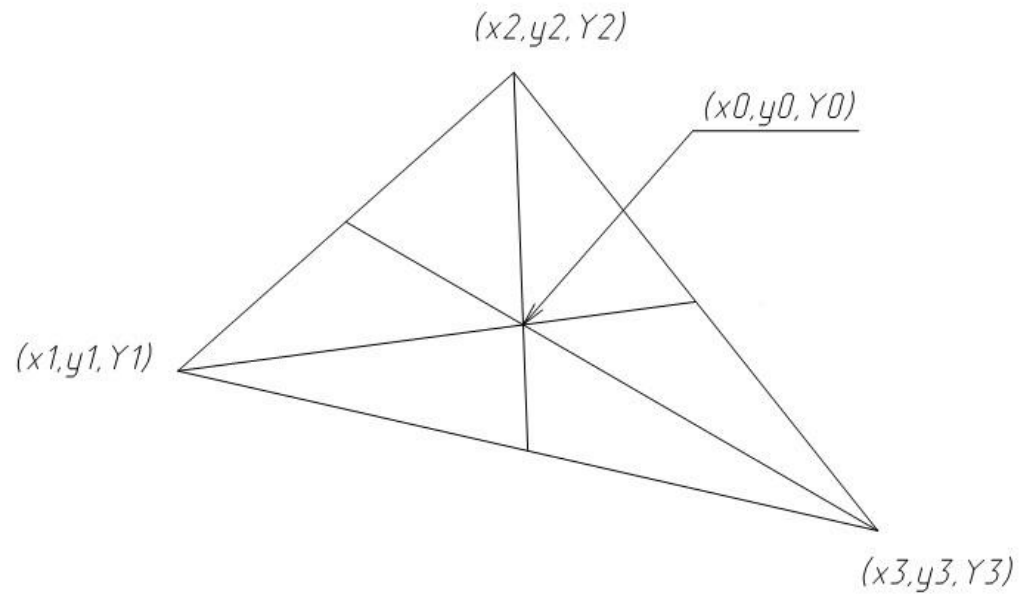

Fig. 2. Forming a color at a point inside the triangle with vertices given by three diffraction points.

The intensity of the diffracted radiation (shown in Fig. 3.) depends on the number of points with diffraction gratings, the more points, the greater the intensity, and, consequently, the brightness of the pixel.

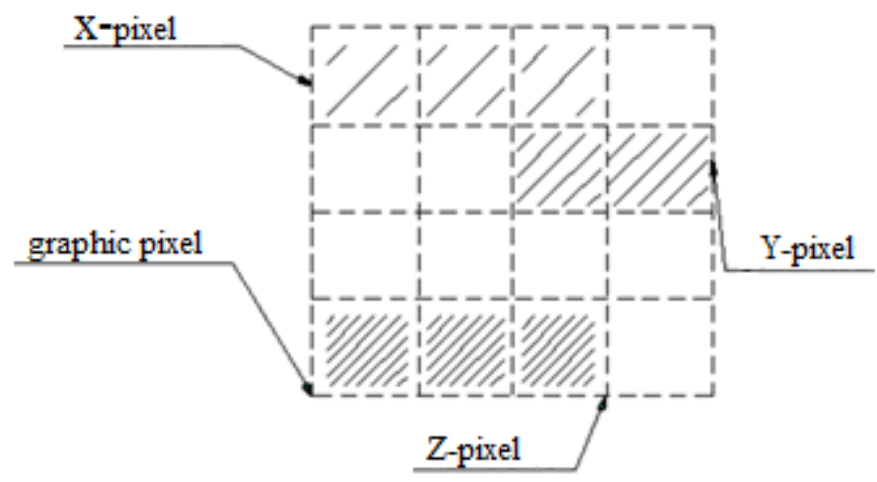

Fig. 3. Schematic representation of a graphic pixel with different intensity of its constituent elements.

The required number of points of each previously defined color depends on the geometric distance in the ICI color chart between the desired color and the three primary colors. In this case, the intensity of the reflected light corresponds to the number of points used to represent the primary color.

\section{Conclusion}

Selecting a sufficient number of diffraction points that create colors close to the border of the ICI color chart, almost all the colors located inside the diagram can be easily display by combining different diffraction pixels. Representation of the image in the ICI colormap system allows to provide a larger color spectrum than RGB.

\section{References}

[1] Magnusson R, Gaylord TK. Diffraction efficiencies of thin phase gratings with arbitrary grating shape. J. Opt. Soc. Am. 1978; 68(6): 87-93.

[2] Frank SD. Holographic image conversion method for making a controlled holographic grating. U.S. patent 5262879 (16 nov. 1993 ).

[3] Kolyuchkin VV, Zlokazov EYu, Odinokov SB, Talalaev VYe, Tsyganov IK. A coherent measurement method for checking the surface microrelief depth in holographic and diffractive optical elements. Computer Optics 2015; 39(4): 515-520. DOI: 10.18287/0134-2452-2015-39-4-515-520.

[4] Khomutov VN, Poleshchuk AG, Cherkashin VV. Measurement of diffraction efficiency of DOE in many diffractive orders. Computer Optics 2011; 35(2): 196-201. 